\title{
Kelvin probe investigations of metal work functions and correlation to device performance of organic light-emitting devices
}

\author{
T.A. Beierlein ${ }^{a, *}$, W. Brütting ${ }^{b}$, H. Riel ${ }^{\text {a }}$, E.I. Haskal ${ }^{\text {a }}$, P. Müller ${ }^{\text {a }}$, W. Rieß ${ }^{\text {a }}$ \\ ${ }^{a}$ IBM Research, Zurich Research Laboratory, CH-8803 Rüschlikon, Switzerland \\ ${ }^{\mathrm{b}}$ Experimentalphysik II, Universität Bayreuth, D-95440 Bayreuth, Germany
}

\begin{abstract}
Using the vibrating capacitor Kelvin probe technique, we have determined the contact potential difference (CPD) between a reference electrode and various metals acting as charge carrier injecting contacts in organic light-emitting devices (OLEDs). These investigations show that the work function of anode materials for OLEDs such as $\mathrm{Pt}, \mathrm{Au}$, and indium tin oxide depends strongly on the surface treatment and can be increased by more than $1 \mathrm{eV}$ via oxygen plasma or UV-ozone cleaning. The device performance of multilayer OLEDs consisting of these anodes, copper-phthalocyanine $(\mathrm{CuPc}), N, N^{\prime}$-di(naphthalene-1-yl)- $N, N^{\prime}$-diphenyl-benzidine (NPB), tris-(8-hydroxyquinolinato)aluminum $\left(\mathrm{Alq}_{3}\right)$, and a low-work-function metal cathode is correlated with the results of the CPD measurements. However, our investigations indicate that, apart from the measured work function, other factors such as the surface roughness and the binding energy of oxygen to the metal surface can significantly influence the injection properties and the long-term stability of the devices.
\end{abstract}

Keywords: Organic light-emitting diode; Kelvin probe; Work function; Anode

\section{Introduction}

Organic light-emitting devices (OLEDs) are a promising technology for highly efficient, lightweight, and lowpower display applications [1]. In recent years, significant progress has been made to increase the rightness, efficiency, and lifetime of such devices [2,3]. For optimized device performance, the injection properties of the electrodes are of crucial importance. By using low-work-function metals/alloys and/or thin inorganic injection layers, electron injection into the organic layer has been significantly improved [4,5]. For efficient hole injection, highwork-function materials are necessary in order to reduce the energy barrier between the Fermi level of the anode and the highest occupied molecular orbital (HOMO) of the adjacent organic layer. Indium tin oxide (ITO), a transparent, degenerate $n$-type semiconductor, is usually used as the anode. On opaque substrates, however, other high-

\footnotetext{
${ }^{*}$ Corresponding author. Tel.: +41-1-724-8321; fax: +41-1-724-8958.

E-mail address: tib@zurich.ibm.com (T.A. Beierlein).
}

work-function materials can be employed, and the light has to be extracted through a transparent top cathode. In order to find materials that are suitable as anodes in OLEDs, the vibrating capacitor Kelvin probe method (KPM) is a powerful tool. This method determines the contact potential difference (CPD) of a sample with respect to a reference electrode and is therefore a relative measure of the work function. The KPM can be performed under ambient conditions, although high vacuum or inert atmosphere is preferred because of the high sensitivity of the CPD to adsorbed layers. The KPM is also useful to monitor changes due to adsorption, desorption or oxidation. Details of the KPM are described elsewhere [6].

In this paper, we present CPD measurements on various metals. We show that UV-ozone and oxygen plasma treatments dramatically change the CPD and its time-dependent behavior. Furthermore, we present first results, which show a correlation between device performance and CPD values.

\section{Experimental}

The metal-coated glass substrates (Schott AF 45) for the Kelvin probe measurements were fabricated in a high- 


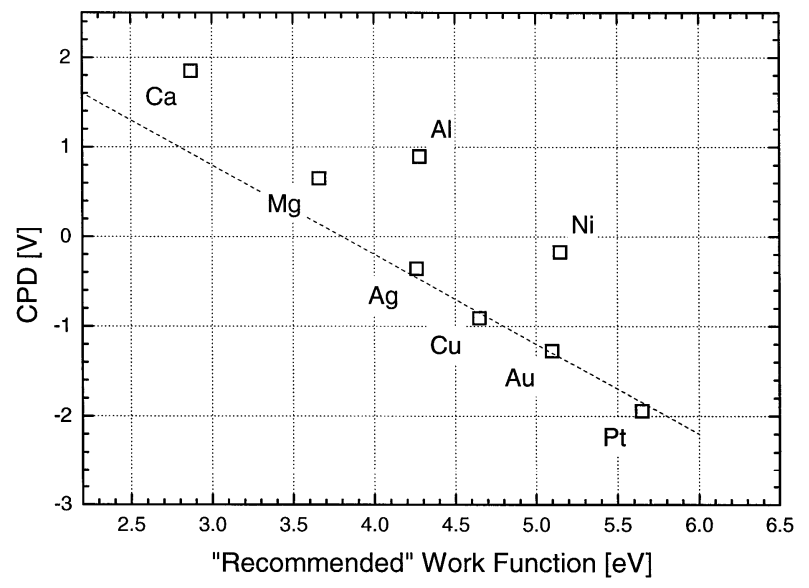

Fig. 1. Comparison of measured CPD values of different metals with their work function reported in the literature.

vacuum $\left(<10^{-7}\right.$ mbar) e-beam evaporator. We used $\mathrm{Ca}$, $\mathrm{Mg}, \mathrm{Ag}, \mathrm{Al}, \mathrm{Cu}, \mathrm{Ni}, \mathrm{Au}$, and $\mathrm{Pt}$ with a typical thickness of $1000 \AA$. In order to reduce surface contamination by oxygen and moisture, the samples were transferred directly after deposition in a vacuum transport case to an argon glove box $\left(\mathrm{O}_{2}<1 \mathrm{ppm}, \mathrm{H}_{2} \mathrm{O}<1 \mathrm{ppm}\right)$. The CPD measurements were performed in the glove box using a McAllister KP6500 digital Kelvin probe, which features a stainless steel tip (diameter $5 \mathrm{~mm}$ ) as the reference electrode. The CPD values are defined as the work function of the tip minus the work function of the sample, i.e., more negative values correspond to a higher work function. The resolution of the system was higher than $5 \mathrm{mV}$. The UV-ozone samples were treated for $1 \mathrm{~h}$ using intense UV light (UVP photo reactor PR-100). The oxygen plasma treatment was performed in a plasma system (TePla 100-E) for $10 \mathrm{~min}$ at a power of $200 \mathrm{~W}$ and an $\mathrm{O}_{2}$ partial pressure of 1 mbar. The reproducibility of the CPD values on different samples is within $0.2 \mathrm{~V}$ for each treatment. X-ray photoelectron spectroscopy (XPS) measurements determined the composition of the treated surfaces using a Sigma Probe (VG Scientific). The multilayer OLEDs were fabricated in a high-vacuum thermal evaporator (base pressure $<5 \times$ $10^{-7}$ mbar), which was connected directly to the argon glove box. The OLEDs consist of copper-phthalocyanine (CuPc, $200 \AA), N, N^{\prime}$-di(naphthalene-1-yl)- $N, N^{\prime}$-diphenylbenzidine (NPB, $470 \AA$ ), tris-(8-hydroxy-quinolinato) aluminum $\left(\mathrm{Alq}_{3}, 650 \AA\right)$ sandwiched between the various anodes and a thin semitransparent low-work-function metal electrode (50 ̊ Ca covered with $140 \AA \mathrm{Mg}: \mathrm{Ag}$ ). To allow a direct comparison of the different anodes, these devices were fabricated simultaneously. The active areas of the OLEDs were $6 \mathrm{~mm}^{2}$. Current-voltage characteristics were taken with a Hewlett Packard Parameter Analyzer HP 4145B, and brightness was measured using a Si photodiode calibrated with a Photo Research PR 704 spectroradiometer.

\section{Results and discussion}

Fig. 1 shows the measured CPD of freshly prepared metal samples plotted vs. their work function values reported in the literature [7]. Ideally, the CPD values should fit onto a line with a slope of -1 in this representation. For $\mathrm{Ag}, \mathrm{Cu}, \mathrm{Au}$, and $\mathrm{Pt}$ this is in good agreement. $\mathrm{Ca}$ and $\mathrm{Mg}$ deviate from this line, which might be because these alkali earths are prone to oxidation and their oxides are reported to have an even lower work function [8]. The deviation of $\mathrm{Al}$ and $\mathrm{Ni}$ from this line cannot be explained by their oxidation and requires further investigation.

In order to determine the critical time scale of changes in the CPD values, we measured the time-dependent behavior and found that the CPD of various metals are stable within a few percent over several hours under argon atmosphere. For example, $\mathrm{Ca}$ with an initial $\mathrm{CPD}$ value of $+1.83 \mathrm{~V}$ changes to $+1.75 \mathrm{~V}$ within $10 \mathrm{~h}$. The most unstable under these conditions is $\mathrm{Au}$, which changes from -1.28 to $-1.12 \mathrm{~V}$, whereas $\mathrm{Pt}$ is the most stable element, its CPD changing only from -1.94 to $-1.93 \mathrm{~V}$ within the same time. However, upon exposing the freshly prepared Pt substrate to ambient conditions, a dramatic change of the CPD from -1.94 to $-0.85 \mathrm{~V}$ is observed after a few minutes. To determine whether these dramatic changes could be reversed, we performed UV-ozone and oxygen plasma treatments on these Pt substrates. After these treatments, we measured values of -2.15 and $-2.24 \mathrm{~V}$ for the UV-ozone and the oxygen plasma-treated Pt samples, respectively. For comparison, the CPD of an oxygen plasma-treated ITO substrate was $-1.95 \mathrm{~V}$. Thus, from a work function point of view, oxygen or UV-ozone-cleaned Pt anodes are expected to show better hole-injecting properties than ITO anodes.

The time-dependent CPD of ozone and oxygen plasmatreated Pt samples is shown in Fig. 2. Both CPD values

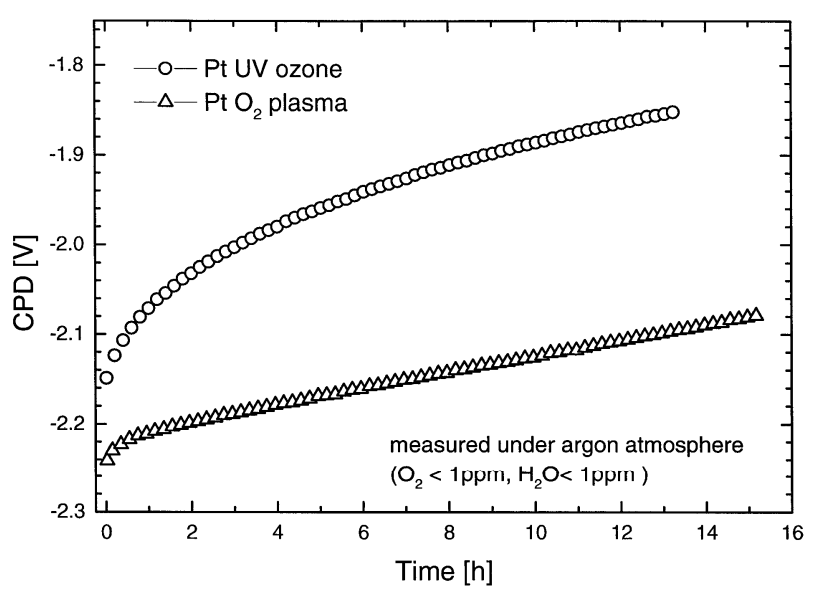

Fig. 2. Contact potential difference over time of UV-ozone and oxygen plasma-treated Pt substrates measured with the KPM under argon atmosphere. 


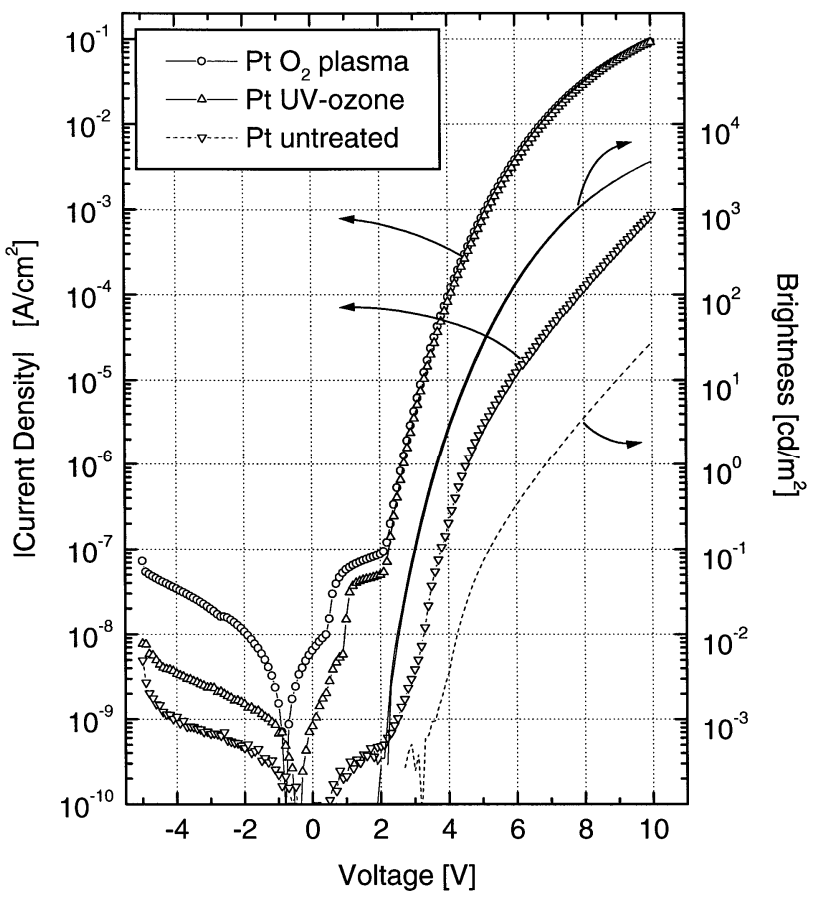

Fig. 3. Current-voltage and brightness-voltage characteristics of multilayer OLEDs fabricated on differently treated Pt anodes.

change significantly towards a lower work function. This process, however, is considerably more pronounced on the UV-ozone-treated Pt sample, indicating a less stable oxide formation or mainly oxygen adsorption. This is supported by preliminary XPS measurements, which show a stronger oxygen 1s signal $(530 \mathrm{eV})$ on the plasma-treated sample.

Fig. 3 depicts the influence of the anode treatment on the device characteristics of anode $/ \mathrm{CuPc} / \mathrm{NPB} / \mathrm{Alq}_{3} /$ $\mathrm{Ca} / \mathrm{Mg}: \mathrm{Ag}$ OLEDs. The different anode treatments resulted in CPD values of $-0.87,-2.22$, and $-2.35 \mathrm{~V}$ for the untreated, the UV-ozone, and the plasma-treated samples, respectively. The OLEDs fabricated onto the plasma and UV-ozone-treated substrates show nearly identical behavior of the $\mathrm{I}-\mathrm{V}$ and electroluminescence-voltage (EL$\mathrm{V})$ characteristics in the forward direction $(U>2 \mathrm{~V})$. The onset of EL in both devices is about $2.1 \mathrm{~V}$; at $10 \mathrm{~V}$ more than $3600 \mathrm{~cd} / \mathrm{m}^{2}$ are achieved at a current density of less than $90 \mathrm{~mA} / \mathrm{cm}^{2}$. This results in an efficiency of $4 \mathrm{~cd} / \mathrm{A}$ measured through a semitransparent metal electrode. The
OLEDs fabricated onto the untreated substrate, however, show relatively poor device performance. The onset of EL is shifted to about $3.5 \mathrm{~V}$, and the current and the brightness in forward direction $(U>2 \mathrm{~V})$ are reduced by more than two orders of magnitude in comparison to the UV-ozone and oxygen plasma-treated samples. Preliminary lifetime studies show a significantly improved device stability of OLEDs built on the plasma-treated $\mathrm{Pt}$ anode in comparison to the untreated and the UV-ozone-treated substrates.

\section{Conclusions}

We showed that Kelvin probe measurements can be used to characterize the bottom electrode of OLEDs before device fabrication. The relative CPD values allow one to make predictions about the injection capabilities of different electrode materials after specific treatments. Furthermore, the time scale of changes in the CPD values can be recorded. The KPM is particularly useful together with atomic force microscopy and XPS in order to identify materials and surface treatments that result in smooth and stable anodes, which are essential for OLEDs with high performance and long-term stability.

\section{Acknowledgements}

We thank Meinrad Tschudy for preparing the metal substrates, and Emmanuel Delamarche for the help with XPS measurements.

\section{References}

[1] C.W. Tang, S.A. VanSlyke, Appl. Phys. Lett. 51 (1987) 913.

[2] Y. Sato, S. Ichinosawa, H. Kanai, IEEE J. Sel. Top. in Quantum Electron. 4 (1998) 40, and references therein.

[3] J. Kido, Y. Iizumi, Appl. Phys. Lett. 73 (1998) 2721.

[4] L.S. Hung, C.W. Tang, M.G. Mason, Appl. Phys. Lett. 70 (1997) 152.

[5] T. Wakimoto, Y. Fukuda, K. Nagayama, A. Yokoi, H. Nakada, M. Tsuchida, IEEE Trans. Electron Devices 44 (1997) 1245.

[6] J.C. Riviere, in: M. Green (Ed.), Solid State Surface Science 1 Marcel Dekker, New York, 1969.

[7] H.B. Michaelson, J. Appl. Phys. 48 (1977) 4729.

[8] V.S. Fomenko, in: V. Samsonov (Ed.), Handbook of Thermionic Properties, Plenum, 1966. 